Published in final edited form as:

Crit Care Med. 2016 February ; 44(2): 360-367. doi:10.1097/CCM.0000000000001572.

\title{
Randomized, Double-Blind, Placebo-Controlled Trial of Thiamine as a Metabolic Resuscitator in Septic Shock: A Pilot Study
}

\author{
Michael W. Donnino, M.D. ${ }^{1,2}$, Lars W. Andersen, M.D. ${ }^{1,3}$, Maureen Chase, M.D., M.P.H. ${ }^{1}$, \\ Katherine M. Berg, M.D. ${ }^{2}$, Mark Tidswell, M.D. ${ }^{4}$, Tyler Giberson, B.S. ${ }^{1}$, Richard Wolfe, M.D. ${ }^{1}$, \\ Ari Moskowitz, M.D. ${ }^{5}$, Howard Smithline, M.D. ${ }^{6}$, Long Ngo, Ph.D. ${ }^{5}$, and Michael N. Cocchi, \\ M.D. ${ }^{1,7}$ for the Center of Resuscitation Science research group \\ ${ }^{1}$ Department of Emergency Medicine, Beth Israel Deaconess Medical Center, Boston, MA, USA \\ ${ }^{2}$ Department of Medicine, Division of Pulmonary and Critical Care, Beth Israel Deaconess \\ Medical Center, Boston, MA, USA \\ ${ }^{3}$ Department of Anesthesiology, Aarhus University Hospital, Aarhus, Denmark \\ ${ }^{4}$ Department of Medicine, Division of Pulmonary and Critical Care, Baystate Medical Center, MA, \\ USA
}

${ }^{5}$ Department of Medicine, Division of General Medicine and Primary Care, Beth Israel Deaconess Medical Center, Boston, MA, USA

${ }^{6}$ Department of Emergency Medicine, Baystate Medical Center, MA, USA

${ }^{7}$ Department of Anesthesia Critical Care, Division of Critical Care, Beth Israel Deaconess Medical Center, Boston, MA, USA

\author{
Abstract \\ shock. \\ Design-Randomized, double-blind, placebo-controlled trial. \\ Setting-Two US hospitals.
}

Objective-To determine if intravenous thiamine would reduce lactate in patients with septic

\footnotetext{
Corresponding Author: Michael W. Donnino, Beth Israel Deaconess Medical Center, Emergency Medicine, One Deaconess Rd, W/CC 2, Boston, MA 02215, P: 617-754-2323, F: 617-754-2350, mdonnino@ bidmc.harvard.edu.

Reprints will not be ordered.

Collaborating Authors from the Center of Resuscitation Science research group

Erin Carney, Julia Balkema, Chris Sulmonte, Justin Salciccioli, Brandon Giberson, Mary MacDonald and Amy Uber.

Copyright form disclosures

Dr. Donnino received support for article research from the National Institutes of Health (NIH), received funding from the National Institute of Alternative and Complementary Medicine (5R21AT005119), and disclosed off-label product use (Thiamine). Dr.

Andersen disclosed off-label product use (Thiamine). Dr. Tidswell received support for article research from the NIH and disclosed off-label product use (Randomized, Double-Blind, Placebo-Controlled Trial of Thiamine as a Metabolic Resuscitator in Septic Shock). His institution received funding from the National Institute for Alternative and Complementary Medicine (distributed to Baystate Medical Center through the primary site which was Beth Israel Hospital). Dr. Ngo received support for article research from the NIH. Dr. Cocchi received support for article research from the NIH. His institution received funding from the NIH. The remaining authors have disclosed that they do not have any potential conflicts of interest.
} 
Patients-Adult patients with septic shock and elevated (> $3 \mathrm{mmol} / \mathrm{L}$ ) lactate between 2010 and 2014.

Interventions-Thiamine $200 \mathrm{mg}$ or matching placebo twice daily for 7 days or until hospital discharge.

Measurements and Main Results-The primary outcome was lactate levels 24 hours after the first study dose. Of 715 patients meeting the inclusion criteria, 88 patients were enrolled and received study drug. There was no difference in the primary outcome of lactate levels at 24 hours after study start between the thiamine and placebo groups (median: $2.5 \mathrm{mmol} / \mathrm{L}[1.5,3.4]$ vs. 2.6 $\mathrm{mmol} / \mathrm{L}[1.6,5.1], \mathrm{p}=0.40$ ). There was no difference in secondary outcomes including time to shock reversal, severity of illness and mortality. $35 \%$ of the patients were thiamine deficient at baseline. In this predefined subgroup, those in the thiamine treatment group had statistically significantly lower lactate levels at 24 hours (median $2.1 \mathrm{mmol} / \mathrm{L}$ [1.4, 2.5] vs. 3.1 [1.9, 8.3], p= $0.03)$. There was a statistically significant decrease in mortality over time in those receiving thiamine in this subgroup $(\mathrm{p}=0.047)$.

Conclusion-Administration of thiamine did not improve lactate levels or other outcomes in the overall group of patients with septic shock and elevated lactate. In those with baseline thiamine deficiency, patients in the thiamine group had significantly lower lactate levels at 24 hours and a possible decrease in mortality over time.

\section{Keywords}

Thiamine; thiamine deficiency; septic shock; sepsis; lactate; metabolism

\section{INTRODUCTION}

Thiamine is an essential vitamin for aerobic metabolism, acting at key junctures for the Krebs Cycle, as well as in the pentose-phosphate shuttle. $(1,2)$ Thiamine deficiency has many clinical manifestations, illustrating the importance of this vitamin. Previously described thiamine deficiency syndromes include Wernicke's Encephalopathy, cardiac beriberi, neuritic beriberi, and gastrointestinal beriberi.(3) Elevated lactate with acidosis is a common manifestation of thiamine deficiency(4-10) and results from failure of oxygen utilization secondary to thiamine's essential role in mitochondrial metabolism.

Thiamine is a key co-factor for pyruvate dehydrogenase, alpha-ketoglutarate dehydrogenase, and transketolase. $(1,2)$ Pyruvate dehydrogenase is the gatekeeper for entry into the Krebs Cycle without which pyruvate will be converted to lactate as opposed to acetyl-coenzyme A. Alpha-ketoglutarate dehydrogenase is required for completion of the Krebs Cycle once it has begun. Transketolase is a key enzyme for the pentose phosphate pathway and the production of NADPH. Thiamine is necessary at each of these three steps. Untreated severe thiamine deficiency (i.e., beriberi) can therefore result in inadequate functioning of aerobic metabolism resulting in elevated lactate, hypotension, and death.(4-16)

Early studies of Wernicke's Encephalopathy found infection was the most common concomitant finding, suggesting that metabolic stress may deplete remaining thiamine stores leading to disease.(2) Several studies have found thiamine deficiency to be prevalent in 
septic shock and other critically ill conditions, with rates ranging from $10 \%$ to $70 \%$ depending on the study design and patient population.(17-21) Elevated lactate, acidosis and hypotension occur in both septic shock and thiamine deficiency. Therefore, a relative or absolute thiamine deficiency state could exist in patients suffering from septic shock. We hypothesized that the provision of thiamine in patients with septic shock would lead to a reduction in lactate. We secondarily hypothesized that this effect would be greater in those with absolute thiamine deficiency.

\section{MATERIALS AND METHODS}

\section{Design and Setting}

This was a two-center, randomized, double-blind, trial comparing thiamine to placebo in patients with septic shock. The study was conducted at Beth Israel Deaconess Medical Center (coordinating site) and Baystate Medical Center, both tertiary care centers in Massachusetts, USA. The study was approved by both Institutional Review Boards, and patients or a legally authorized surrogate provided written informed consent. The trial was registered at clinicaltrials.gov (NCT01070810). The Data and Safety Monitoring Board evaluated and monitored for safety; efficacy and futility stopping points were not considered given the pilot nature of the study.

\section{Study Population}

The hospitals' emergency departments and intensive care units (ICU) were screened between January 2010 and October 2014. Inclusion criteria were: age $\geq 18$ years, sepsis (presence of two or more systemic inflammatory response syndrome (SIRS) criteria with documented or suspected infection), lactate $>3 \mathrm{mmol} / \mathrm{L}$ (at the time of informed consent and randomization), and hypotension (systolic pressure $<90 \mathrm{mmHg}$ ) after $\geq 2 \mathrm{~L}$ fluid bolus followed by vasopressor-dependence. Vasopressor-dependence was defined as the continuous infusion of norepinephrine, dopamine ( $25 \mathrm{mcg} / \mathrm{kg} / \mathrm{min}$ ), phenylephrine, vasopressin (>0.04 units/min) or epinephrine. Dobutamine or other inotropes at any dose were not considered vasopressors.

Exclusion criteria included: 1) liver injury or dysfunction (aspartate transaminase or alanine transaminase $>240$ units/L or known cirrhosis) based on previous findings that patients with liver dysfunction may have elevated thiamine levels(17), 2) current thiamine supplementation, 3) clinical indication for thiamine as judged by the clinical team (e.g., alcohol abuse), 4) "comfort measures only" (CMO) designation, 5) inability to provide consent, and 6) competing cause(s) of elevated lactate levels (including seizure within 3 hours, medications associated with elevated lactate (i.e. metformin, linezolid and antiretrovirals), carbon monoxide or cyanide poisoning, known mitochondrial disorder, known/ suspected bowel or limb ischemia, or cardiac arrest prior to enrollment).(17, 22, 23) Enrollment could occur at any time during the hospitalization.

\section{Randomization, Study Drug and Blinding}

Patients were randomized to thiamine or placebo in a 1:1 ratio stratified by site. Patients received $200 \mathrm{mg}$ thiamine in $50 \mathrm{~mL} 5 \%$ dextrose or matching placebo (50 $\mathrm{mL} 5 \%$ dextrose) 
twice daily for 7 days or until hospital discharge. Placebo was identical in appearance to thiamine and patients, healthcare personnel and the research team remained blinded throughout the study period.

\section{Blood Samples and Data Collection}

Blood was collected at enrollment before $(<10 \mathrm{~min}$ ) administration of the study drug (time 0 ) and at 6,12 and 24 hours thereafter ( \pm 1 hour). Blood was sent for a lactate measurement at the hospitals' clinical laboratories. Samples were centrifuged, aliquoted into lightprotected cryotubes, and frozen at $-80{ }^{\circ} \mathrm{C}$. Thiamine levels were measured in plasma via Liquid Chromatography/Tandem Mass Spectrometry by Quest Diagnostics (Nichols Institute, Chantilly, VA, USA). Absolute thiamine deficiency ( $\leq 7 \mathrm{nmol} / \mathrm{L}$ ) was determined using a previously established standard laboratory reference range.

\section{Outcome Measures}

The primary outcome was lactate level 24 hours after the first study medication dose. Secondary outcomes included lactate levels at 6 and 12 hours as well as lactate change at 24 hours (defined as (lactate at 0 hour - lactate at 24 hour)/lactate at 0 hour $\times 100 \%$ ) and lactate $\geq 4 \mathrm{mmol} / \mathrm{L}$ at 24 hours (a commonly used cutoff indicating a high lactate level). $(22,23)$ Additional outcomes included time to shock reversal ( $>24$ hours off all vasopressors), APACHE II score(24) at 24 hours, SOFA score(25) at 24 hours, ICU and hospital length of stay, and inhospital mortality.

Among those who died, we classified the mode of death into one of the following categories: sudden cardiac arrest, hemodynamic shock, respiratory failure, neurological or co-morbid withdrawal of care, or "other" based on predefined criteria. The assessment of all clinical outcomes was performed by members of the investigative team who were blinded to the treatment group allocations.

\section{Power Calculation and Statistical Analyses}

The sample size was calculated based on relative lactate change at 24 hours. Preliminary data suggested control patients would have a relative reduction of $50 \%$ with a standard deviation (SD) of $26 \%$. We anticipated that the treatment group would have a relative reduction of $67 \%$ with a similar SD. With a type-I error of 0.05 , and power of 0.80 , we estimated the need for 80 patients. This estimate was increased by $10 \%$ to ensure an adequate sample size for analysis of patients with thiamine deficiency. Although the power calculation was for relative change, our primary endpoint focused on the absolute $24 \mathrm{~h}$ lactate based on input from the Food and Drug Administration (FDA) after the trial had begun. The change in the final analysis was approved through the DSMB prior to unblinding.

Descriptive statistics were used to summarize the study population. Continuous variables are presented as means (SD) or medians $\left(1^{\text {st }}\right.$ quartile, $3^{\text {rd }}$ quartile) depending on the normality of the data. Categorical data are presented as counts with frequencies and compared between groups using Fisher's exact tests. Depending on the distribution of the data, t-tests or Wilcoxon rank sum tests were used to compare continuous data between the groups. All 
analysis was based on a modified intention to treat principle where all patients who received at least one dose of the study drug were included.(26) For the 24 hour time point, lactate levels were imputed based on a pre-defined plan in patients who died before this time point. If patients died before the 6 hour time point (five patients) a 20\% increase from baseline was imputed, if patients died between the 6 and 12 hour time point (one patient) a 15\% increase from the 6 hour time point was imputed and if patients died between the 12 and 24 hour time point (six patients) a $10 \%$ increase from the 12 hour time point was imputed.

As a pre-defined secondary analysis, we assessed the difference in lactate levels between groups over time. We utilized a repeated measures model including treatment group (thiamine and placebo), a within-subject factor (time: 0, 6, 12, and 24 hours) and the interaction between these two. An autoregressive covariance structure provided the best model fit based on the Akaike Information Criterion (AIC). Given the right-skewed distribution of the lactate levels they were log-transformed before entry in the mixed linear model and non-imputed values were used for this analysis.

Time to shock reversal was complicated by a high incidence of death prior to the event. To account for this we classified death as a competing risk event and used the estimated cumulative incidence function (CIF) to illustrate the comparison of CIFs between the two treatment groups using the Fine-Gray competing risk model.(27) We tested the subdistribution hazards of these two CIF functions and obtained the estimated hazard ratio with $95 \%$ confidence intervals. Kaplan Meier curves were created for survival and compared with the log-rank test. Patients were censored at hospital discharge.

Pre-planned sub-analyses were performed in the thiamine deficient patients at the coordinating site. All hypothesis tests were two-sided, with a significance level of $\mathrm{p}<0.05$. No adjustments were made for multiple testing and all secondary outcomes should therefore be considered exploratory. Statistical analyses were conducted with the use of SAS software, version 9.4 (SAS Institute Inc., Cary, NC, USA).

\section{RESULTS}

\section{Patient Characteristics}

Of 715 patients meeting inclusion criteria, 88 were enrolled and received the first study dose (see Figure 1); 80 patients were enrolled at the coordinating site. The mean age was 67 years (SD: 16), 36 (41\%) were female, and in-hospital mortality was $43 \%$. Forty-three patients received thiamine and 45 received placebo with baseline characteristics well-balanced between the groups although patients in the thiamine group appeared to have more noninsulin dependent diabetes (Table 1).

\section{Lactate}

There was no difference in the primary outcome of lactate levels at 24 hours (median: 2.5 $\mathrm{mmol} / \mathrm{L}[1.5,3.4]$ vs. $2.6 \mathrm{mmol} / \mathrm{L}[1.6,5.1], \mathrm{p}=0.40$, Figure 2$)$. In the repeated measures model there were statistically significantly lower lactate levels in the thiamine group at 24 hours ( $p=0.048$, Figure 2). Relative lactate change from baseline to 24 hours was $42 \%$ ( 17 , $51)$ in the thiamine group and $25 \%(-12,47)$ in the placebo group $(p=0.16)$. Seventeen 
patients (38\%) had a lactate $\geq 4 \mathrm{mmol}$ at 24 hours in the placebo group compared to 9 (21\%) in the thiamine group $(\mathrm{p}=0.10)$. There was no difference in lactate between groups at 6 hours $(\mathrm{p}=0.62)$ or 12 hours $(\mathrm{p}=0.44)$.

\section{Secondary Outcomes}

We found no difference in the proportion of patients with shock reversal between the thiamine and placebo groups $(74 \%$ vs. $71 \%, \mathrm{p}=0.81)$. In the competing risk analysis we found no difference in time to shock reversal between the thiamine and placebo groups (hazard ratio: 0.99 [95\% CI: $0.61-1.62], \mathrm{p}=0.97$ ).

The APACHE II score at 24 hours was 26 (SD: 10) in the placebo group and 23 (SD: 8) in the thiamine group $(\mathrm{p}=0.15)$. The 24 hour SOFA score was 8.9 (SD: 5.0$)$ in the placebo group and 8.1 (SD: 3.5$)$ in the thiamine group $(\mathrm{p}=0.41$ ). Time to ICU discharge and hospital length of stay in survivors were similar in those receiving placebo and thiamine (7 days $[3,18]$ vs. 8 days $[4,13], \mathrm{p}=0.70)$ and $(13$ days $[7,24)$ vs. 13 days $[8,20], \mathrm{p}=0.86)$. Inpatient mortality was also similar in both groups ( $42 \mathrm{vs} 44 \%, \mathrm{p}=1.00)$. There was no difference between the survival curves for the overall group ( $p=0.85$, Figure 3 ) or in the mode of death $(\mathrm{p}=0.51$, Table 2$)$.

\section{Patients with Baseline Thiamine Deficiency}

We measured baseline thiamine levels in 79 patients. Of these, $28(35 \%)$ were thiamine deficient ( $\leq 7 \mathrm{nmol} / \mathrm{L}$ ); 15 in the thiamine group and 13 in the placebo group. Among thiamine deficient patients, those in the thiamine group had statistically significantly lower lactate levels at 24 hours (median $2.1 \mathrm{mmol} / \mathrm{L}[1.4,2.5]$ vs. 3.1 [1.9, 8.3], $\mathrm{p}=0.03$ ). The relative lactate change was $44 \%(29 \%, 49 \%)$ in the thiamine group and $20 \%(-20 \%, 47 \%)$ in the placebo group $(\mathrm{p}=0.18)$. One $(7 \%)$ patient in the thiamine group had a 24 hour lactate level $\geq 4 \mathrm{mmol} / \mathrm{L}$ compared to $5(38 \%)$ patients in the placebo group $(\mathrm{p}=0.07)$. In the repeated measures model there were statistically significantly lower lactate levels in the thiamine group at 24 hours $(p=0.006$, Figure 2$)$. There was no statistically significant difference in lactate levels at $6(\mathrm{p}=0.12)$ or 12 hours $(\mathrm{p}=0.10)$. Individual lactate levels are displayed in eFigure 1 (supplemental material). In the thiamine group, 2 (13\%) patients died compared to $6(46 \%)$ patients in the placebo group $(\mathrm{p}=0.10)$. There was a statistically significant difference between the survival curves $(p=0.047$, Figure 3$)$. There was no difference in the mode of death between groups $(\mathrm{p}=1.00$, Table 2$)$.

\section{DISCUSSION}

For the group overall, we did not detect a difference in lactate levels at 24 hours, although we did find a statistically significant difference in decrease in lactate from baseline to 24 hours. We did not find any difference between groups in clinical parameters including shock reversal, survival, and length of stay. In patients who were thiamine deficient, there was a statistically significantly lower lactate at 24 hours and a decrease in lactate over time in the group who received thiamine compared to placebo. In addition, there was a statistically significant difference in time to death between the groups. 
While our primary hypothesis was that thiamine would be effective for the overall group, we secondarily hypothesized that it would be particularly effective for patients with thiamine deficiency. Patients with thiamine deficiency can present with a range of dysfunctions with neurologic and cardiovascular manifestations predominating. Profound lactate elevation with acidosis has been reported in not only classic cardiac beriberi but also in Wernicke's Encephalopathy and other thiamine deficiency syndromes. Predisposition to thiamine deficiency can come from a myriad of conditions that are associated with nutritional compromise.(3, 28-35). Many of these predisposing factors may also be present in patients with septic shock. In addition to a predisposition to deficiency from underlying co-morbid disease, patients with septic shock have increased metabolic demand and often decreased nutritional intake that could theoretically deplete thiamine levels further. Prior work has shown that thiamine levels are decreased with the stress of cardiac surgery.(36, 37) Finally, Wernicke's Encephalopathy is often precipitated by an underlying stressor. In one series, concurrent infection was reported in upwards of $60 \%$ of cases of the Wernicke-Korsakoff Syndrome.(2) Septic patients may be predisposed to deficiency prior to onset of the illness and may also develop deficiency after illness onset.

In the present study, $35 \%$ of patients had thiamine deficiency at baseline. The percent of deficient patients was higher than in our previous publication in septic shock(17), likely explained by the inclusion and exclusion criteria in the current study. We targeted a population with a high risk for mortality and an increased likelihood of thiamine deficiency. For example, all patients enrolled were required to be on vasopressors with a persistent lactate $>3 \mathrm{mmol} / \mathrm{L}$ after 2 liters of intravenous fluids. In addition, we excluded patients with liver injury to avoid enrolling patients with another cause for decreased capacity to metabolize lactate and because our preliminary data illustrated that patients with acute liver failure often had high thiamine levels.(17) For all these reasons, our selected population formed a high-risk group for thiamine deficiency. At the same time, we did not include the traditional "at-risk" population of alcoholics because these patients would receive thiamine for alcoholism to prevent Wernicke's Encephalopathy as part of standard care. If alcoholics were included, the possibility remains that both the incidence of deficiency and the treatment effect of thiamine would have been increased.

Among thiamine deficient patients, we found both clinical and metabolic differences between the treatment and placebo groups. Lactate levels were lower at 24 hours in the thiamine group compared to placebo, and mortality was improved over time (Figure 3 ). The sample size for this group was small, and future studies will be necessary to validate these findings. That thiamine would decrease lactate in deficiency states is consistent with the expected pathophysiology and suggests that a sub-clinical form of beriberi may exist in patients with septic shock. Septic shock can have manifestations similar to cardiac beriberi (hypotension, hyperdynamic state, elevated lactate, acid-base patterns) and thiamine levels are not typically assessed in this disease state, therefore setting up the potential for unrecognized concomitant beriberi. Concomitant beriberi in septic shock could theoretically be even more prominent in developing countries with higher rates of nutritional deficiencies.

The mortality in the current trial was higher than recently reported studies in septic shock(38-40), but this is likely reflective of differences in the inclusion/exclusion criteria. A 
recent, large study from the Surviving Sepsis database reveals that mortality rates among those with hypotension and elevated lactate (i.e. the inclusion criteria in this study) were similar to the current trial.(41)

The current trial has several limitations. Enrollments were predominately from one site and generalizability may be limited. The sample size for the thiamine deficient group was small $(n=28)$ and therefore the results for this population need to be reproduced. Our inclusion and exclusion criteria likely led to a cohort with a high rate of deficiency. Therefore, the rate of deficiency and the potential therapeutic effect of thiamine in the overall septic population remain unknown. We did not exclude those on epinephrine, which may cause elevated lactate(22) (although this drugs was used in $<10 \%$ of patients). The trial design did not specify a maximum time for enrollment and the possibility remains that the treatment effect of thiamine may have some time-dependence. Finally, while we based our dosage on historical dosages in other disease states and dosages that effectively resolved lactate elevation in cases of pure thiamine deficiency, we did not perform a dose-finding trial, and therefore the possibility remains that other regimens would be more effective.

\title{
CONCLUSION
}

In a randomized trial of intravenous thiamine for patients in septic shock, we did not find a difference in lactate levels between groups at 24 hours. We did, however, find a statistically significant decrease in lactate from baseline to 24 hours in the thiamine group compared to the placebo group. There were no differences in clinical outcomes. In the sub-set of patients with thiamine deficiency, we found decreased lactate at 24 hours and a possible decrease in mortality over time in the thiamine group compared to the placebo group. Future studies are needed, particularly targeting the deficient group.

\section{Supplementary Material}

Refer to Web version on PubMed Central for supplementary material.

\section{Acknowledgments}

\author{
Funding \\ This study was funded by a grant from the National Institute of Alternative and Complementary Medicine \\ (5R21AT005119).
}

The authors wish to thank Francesca Montillo for her administrative support for the trial. The authors wish to thank the Data and Safety Monitoring Board Members including Dr. Derek Angus, Dr. Joseph Miller, and Dr. Curtis Sessler as well as the independent statistical and investigative oversight by Teresa Nelson and Dr. Florence Bourgeois

\section{References}

1. Frank RA, Leeper FJ, Luisi BF. Structure, mechanism and catalytic duality of thiamine-dependent enzymes. Cell Mol Life Sci. 2007; 64(7-8):892-905. [PubMed: 17429582]

2. Victor, M.; Adams, R.; Collins, G. The Wernicke-Korsakoff Syndrome and Related Neurologic Disorders Due to Alcoholism and Malnutrition. 2. Quest-Meridien Ltd; 1989. 
3. Donnino MW, Vega J, Miller J, et al. Myths and misconceptions of Wernicke's encephalopathy: what every emergency physician should know. Ann Emerg Med. 2007; 50(6):715-721. [PubMed: 17681641]

4. Chisolm-Straker M, Cherkas D. Altered and unstable: wet beriberi, a clinical review. J Emerg Med. 2013; 45(3):341-344. [PubMed: 23849362]

5. Rao SN, Chandak GR. Cardiac beriberi: often a missed diagnosis. J Trop Pediatr. 2010; 56(4):284285. [PubMed: 19934228]

6. Kitamura K, Yamaguchi T, Tanaka H, et al. TPN-induced fulminant beriberi: a report on our experience and a review of the literature. Surg Today. 1996; 26(10):769-776. [PubMed: 8897674]

7. Betrosian AP, Thireos E, Toutouzas K, et al. Occidental beriberi and sudden death. Am J Med Sci. 2004; 327(5):250-252. [PubMed: 15166742]

8. Attas M, Hanley HG, Stultz D, et al. Fulminant beriberi heart disease with lactic acidosis: presentation of a case with evaluation of left ventricular function and review of pathophysiologic mechanisms. Circulation. 1978; 58(3 Pt 1):566-572. [PubMed: 679449]

9. Kountchev J, Bijuklic K, Bellmann R, et al. A patient with severe lactic acidosis and rapidly evolving multiple organ failure: a case of shoshin beri-beri. Intensive Care Med. 2005; 31(7):1004. [PubMed: 15875157]

10. Panichewa S, Hathirat S. Fulminant cardiac beriberi with severe acidosis. J Med Assoc Thai. 1982; 65(10):566-569. [PubMed: 7175406]

11. Manzanares W, Hardy G. Thiamine supplementation in the critically ill. Curr Opin Clin Nutr Metab Care. 2011; 14(6):610-617. [PubMed: 21912244]

12. Thauvin-Robinet C, Faivre L, Barbier ML, et al. Severe lactic acidosis and acute thiamin deficiency: a report of 11 neonates with unsupplemented total parenteral nutrition. J Inherit Metab Dis. 2004; 27(5):700-704. [PubMed: 15669689]

13. Kleiner-Baumgarten A, Sidi A, Abu-Shakra M, et al. Thiamine deficiency among Chinese workers in Israel. Harefuah. 2003; 142(5):329-331. 400, 399. [PubMed: 12803052]

14. Busani S, Bonvecchio C, Gaspari A, et al. Wernicke's encephalopathy in a malnourished surgical patient: a difficult diagnosis. BMC Res Notes. 2014; 7:718. [PubMed: 25312751]

15. Campos L, Masclans JR, Ortega A, et al. Neurological disturbances and hyperdynamic shock in a patient with esophagocoloplasty. Intensive Care Med. 1997; 23(5):590-592. [PubMed: 9201534]

16. Campbell $\mathrm{CH}$. The severe lacticacidosis of thiamine deficiency: acute pernicious or fulminating beriberi. Lancet. 1984; 2(8400):446-449. [PubMed: 6147512]

17. Donnino MW, Carney E, Cocchi MN, et al. Thiamine deficiency in critically ill patients with sepsis. J Crit Care. 2010; 25(4):576-581. [PubMed: 20646908]

18. Lima LF, Leite HP, Taddei JA. Low blood thiamine concentrations in children upon admission to the intensive care unit: risk factors and prognostic significance. Am J Clin Nutr. 2011; 93(1):5761. [PubMed: 21068344]

19. Seear M, Lockitch G, Jacobson B, et al. Thiamine, riboflavin, and pyridoxine deficiencies in a population of critically ill children. J Pediatr. 1992; 121(4):533-538. [PubMed: 1403385]

20. Cruickshank AM, Telfer AB, Shenkin A. Thiamine deficiency in the critically ill. Intensive Care Med. 1988; 14(4):384-387. [PubMed: 3136196]

21. Costa NA, Gut AL, de Souza Dorna M, et al. Serum thiamine concentration and oxidative stress as predictors of mortality in patients with septic shock. J Crit Care. 2014; 29(2):249-252. [PubMed: 24412011]

22. Andersen LW, Mackenhauer J, Roberts JC, et al. Etiology and therapeutic approach to elevated lactate levels. Mayo Clin Proc. 2013; 88(10):1127-1140. [PubMed: 24079682]

23. Donnino MW, Andersen LW, Giberson T, et al. Initial lactate and lactate change in post-cardiac arrest: a multicenter validation study. Crit Care Med. 2014; 42(8):1804-1811. [PubMed: 24776606]

24. Knaus WA, Draper EA, Wagner DP, et al. APACHE II: a severity of disease classification system. Crit Care Med. 1985; 13(10):818-829. [PubMed: 3928249]

25. Vincent JL, Moreno R, Takala J, et al. The SOFA (Sepsis-related Organ Failure Assessment) score to describe organ dysfunction/failure. On behalf of the Working Group on Sepsis-Related 
Problems of the European Society of Intensive Care Medicine. Intensive Care Med. 1996; 22(7): 707-710. [PubMed: 8844239]

26. Fergusson D, Aaron SD, Guyatt G, et al. Post-randomisation exclusions: the intention to treat principle and excluding patients from analysis. BMJ. 2002; 325(7365):652-654. [PubMed: 12242181]

27. Fine J, Gray R. A proportional hazards model for the subdistribution of a competing risk. Journal of the American Statistical Association. 1999; 94:496-509.

28. Moskowitz A, Graver A, Giberson T, et al. The relationship between lactate and thiamine levels in patients with diabetic ketoacidosis. J Crit Care. 2014; 29(1):182e185-188. [PubMed: 23993771]

29. Thornalley PJ, Babaei-Jadidi R, Al Ali H, et al. High prevalence of low plasma thiamine concentration in diabetes linked to a marker of vascular disease. Diabetologia. 2007; 50(10):21642170. [PubMed: 17676306]

30. Boniol S, Boyd M, Koreth R, et al. Wernicke encephalopathy complicating lymphoma therapy: case report and literature review. South Med J. 2007; 100(7):717-719. [PubMed: 17639753]

31. Lakhani SV, Shah HN, Alexander K, et al. Small intestinal bacterial overgrowth and thiamine deficiency after Roux-en-Y gastric bypass surgery in obese patients. Nutr Res. 2008; 28(5):293298. [PubMed: 19083422]

32. Kotha VK, De Souza A. Wernicke's encephalopathy following Hyperemesis gravidarum. A report of three cases. Neuroradiol J. 2013; 26(1):35-40. [PubMed: 23859165]

33. Wierzbicka-Chmiel J, Wierzbicki K, Kajdaniuk D, et al. Wernicke-Korsakoff syndrome in the course of thyrotoxicosis - a case report. Endokrynol Pol. 2011; 62(2):178-180. [PubMed: 21528481]

34. Renthal W, Marin-Valencia I, Evans PA. Thiamine deficiency secondary to anorexia nervosa: an uncommon cause of peripheral neuropathy and Wernicke encephalopathy in adolescence. Pediatr Neurol. 2014; 51(1):100-103. [PubMed: 24938142]

35. Giacalone M, Martinelli R, Abramo A, et al. Rapid reversal of severe lactic acidosis after thiamine administration in critically ill adults: a report of 3 cases. Nutr Clin Pract. 2015; 30(1):104-110. [PubMed: 25516536]

36. Donnino MW, Cocchi MN, Smithline H, et al. Coronary artery bypass graft surgery depletes plasma thiamine levels. Nutrition. 2010; 26(1):133-136. [PubMed: 20005469]

37. Kim WY, Kwak MK, Ko BS, et al. Factors associated with the occurrence of cardiac arrest after emergency tracheal intubation in the emergency department. PLoS One. 2014; 9(11):e112779. [PubMed: 25402500]

38. Investigators A, Group ACT, Peake SL, et al. Goal-directed resuscitation for patients with early septic shock. N Engl J Med. 2014; 371(16):1496-1506. [PubMed: 25272316]

39. Mouncey PR, Osborn TM, Power GS, et al. Trial of early, goal-directed resuscitation for septic shock. N Engl J Med. 2015; 372(14):1301-1311. [PubMed: 25776532]

40. Yealy DM, Kellum JA, et al. Process Investigators. A randomized trial of protocol-based care for early septic shock. N Engl J Med. 2014; 370(18):1683-1693. [PubMed: 24635773]

41. Casserly B, Phillips GS, Schorr C, et al. Lactate measurements in sepsis-induced tissue hypoperfusion: results from the Surviving Sepsis Campaign database. Crit Care Med. 2015; 43(3): 567-573. [PubMed: 25479113] 
715 patients met inclusion criteria

(Sepsis, shock, and lactate $>3 \mathrm{mmol} / \mathrm{L}$ )

\section{Excluded:}

Liver dysfunction $(n=212)$

Comfort measure only $(n=79)$

Other reasons $(n=66)$

Thiamine use $(n=56)$

Metformin, linezolid, or anti-retroviral $(n=55)$

Refused participation $(n=48)$

No family present to consent $(n=27)$

Cardiac arrest $(n=16)$

Bowel or limb ischemia $(n=16)$

Seizures within 3 hours $(n=14)$

Enrolled but subsequently did not meet inclusion criteria $(n=13)$

Enrolled in other interventional trial $(n=11)$

Protected population $(n=4)$

Non-English speaking $(n=4)$

Previously enrolled $(n=2)$

92 patients randomized

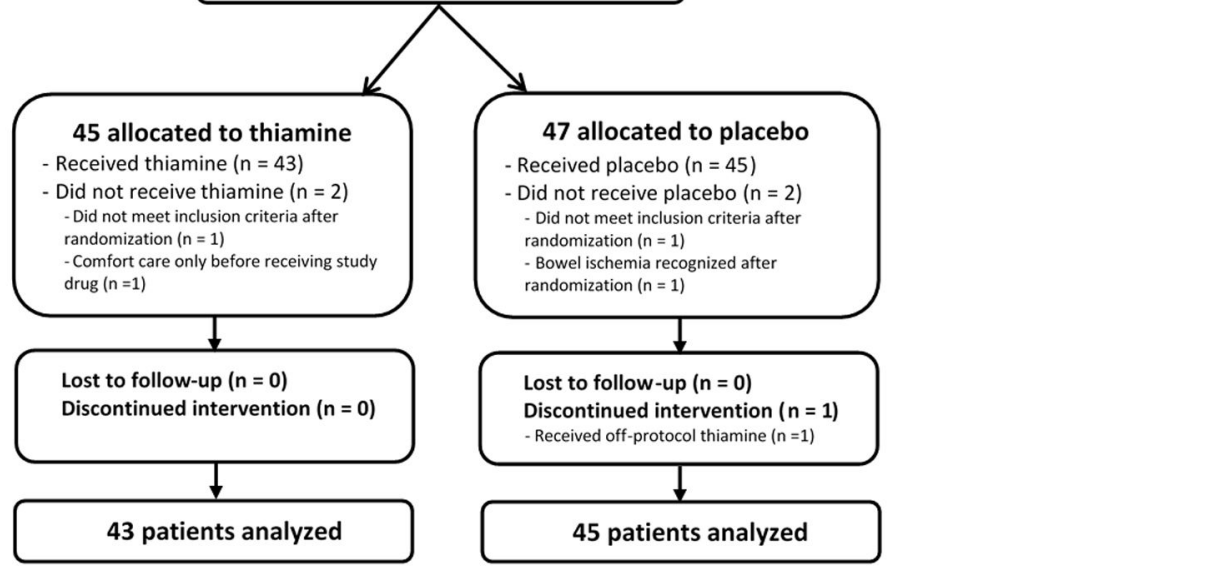

Figure 1. CONSORT flow diagram

Out of 715 patients with septic shock and elevated lactate, 88 were included in the analysis. 


$$
\text { Complete group }(n=88)
$$

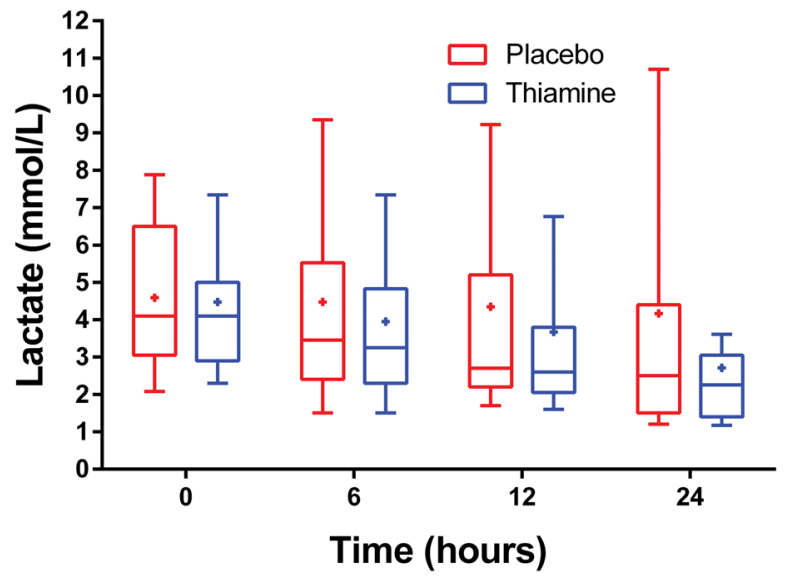

Thiamine deficient group $(\mathbf{n}=\mathbf{2 8})$

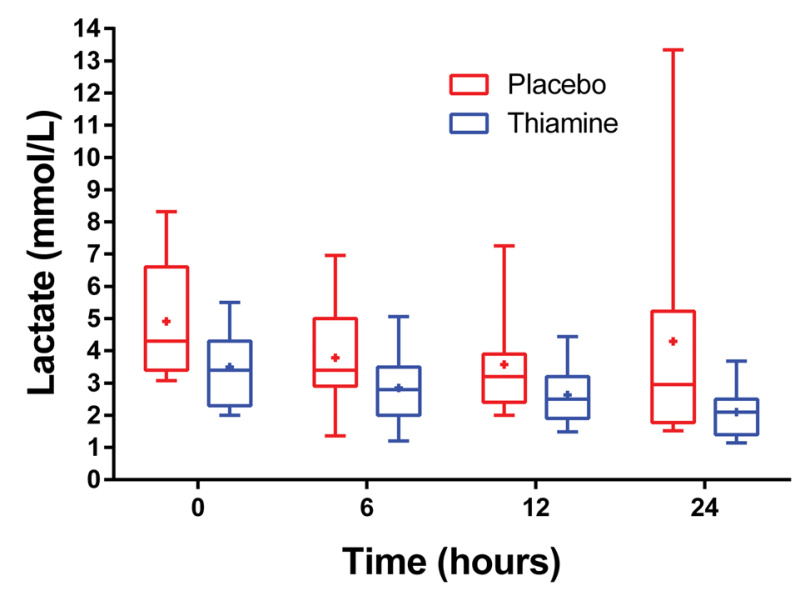

Figure 2. Lactate levels over time

Lactate levels at baseline (time of study drug) and 6, 12 and 24 hours thereafter according to treatment group in the full study group (left) and in the thiamine deficient cohort (right). The boxplots represent the $1^{\text {st }}$ quartiles, median, and $3^{\text {rd }}$ quartile. The whiskers represent the $10^{\text {th }}$ and $90^{\text {th }}$ percentile and the " + " is the mean. 
Complete group $(n=88)$

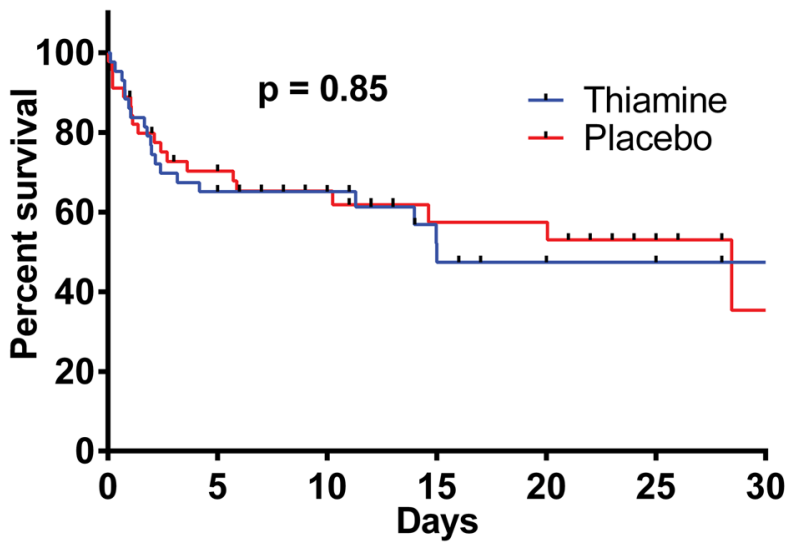

Thiamine deficient group $(n=28)$

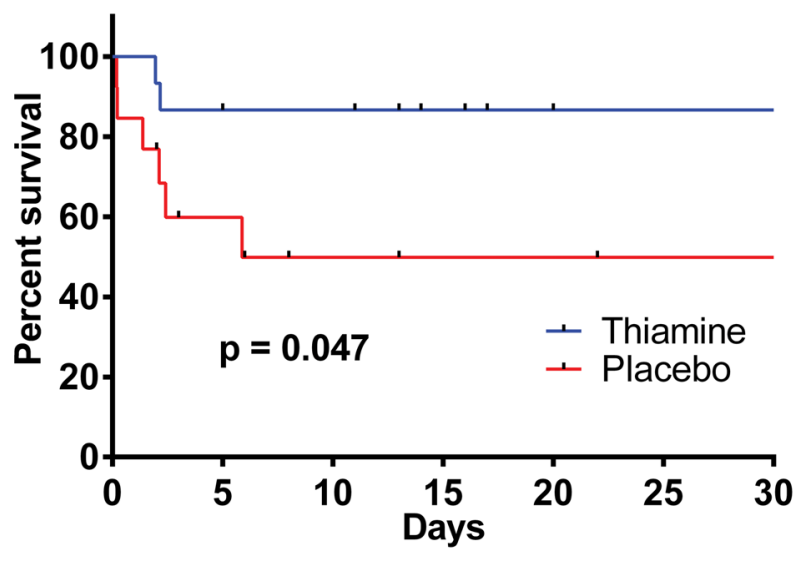

Figure 3. Kaplan Meier survival curves

Survival curves for the thiamine and placebo groups in the full study group (left) and the thiamine deficient group (right). Patients were censored at hospital discharge. The graph is truncated at 30 days for illustrative purposes. Vertical lines represents censored patients and the p-value is from the log-rank test. 


\section{Table 1}

Selected Baseline Characteristics of the Study Patients ${ }^{a}$

\begin{tabular}{|c|c|c|}
\hline & Thiamine $(n=43)$ & Placebo $(n=45)$ \\
\hline \multicolumn{3}{|l|}{ Demographics } \\
\hline Age - mean years $(\mathrm{SD})$ & $70(14)$ & $65(17)$ \\
\hline Sex - no. female (\%) & $17(40)$ & $19(42)$ \\
\hline Race - no. white (\%) & $36(84)$ & $40(89)$ \\
\hline $\mathrm{BMI}^{b}-\mathrm{kg} / \mathrm{m}^{2}(\mathrm{SD})$ & $29(9)$ & $29(7)$ \\
\hline \multicolumn{3}{|l|}{ Co-morbidities - No. (\%) } \\
\hline Coronary artery disease & $9(21)$ & $10(22)$ \\
\hline Congestive heart failure & $10(23)$ & $12(27)$ \\
\hline Hypertension & $24(56)$ & $22(49)$ \\
\hline Chronic pulmonary disease & $10(23)$ & $13(29)$ \\
\hline Diabetes & $19(44)$ & $6(13)$ \\
\hline Insulin-dependent & $5(12)$ & $5(11)$ \\
\hline Charlson Comorbidity Index ${ }^{c}-$ median (quartiles) & $2(1,3)$ & $3(1,5)$ \\
\hline \multicolumn{3}{|l|}{ Lactate values } \\
\hline Lactate - median mmol/L (quartiles) & $4.1(2.9,5.0)$ & $4.1(3.1,6.4)$ \\
\hline Lactate $>4 \mathrm{mmol} / \mathrm{L}-$ no. $(\%)$ & $26(60)$ & $24(53)$ \\
\hline \multicolumn{3}{|l|}{ Laboratory values at enrollment ${ }^{d}-$ median (quartiles) } \\
\hline White blood count $\left(\times 10^{3}\right)$ & $13.9(8.7,22.5)$ & $13.1(4.6,19.3)$ \\
\hline Hemoglobin $(\mathrm{g} / \mathrm{dL})$ & $10.0(8.9,13.0)$ & $10.4(9.3,12.5)$ \\
\hline Creatinine (mg/dL) & $1.8(1.0,2.6)$ & $1.9(1.4,3.0)$ \\
\hline Glucose (mg/dL) & $145(91,191)$ & $136(116,191)$ \\
\hline \multicolumn{3}{|l|}{ Mechanical ventilation and severity of illness } \\
\hline Mechanical ventilation at time of enrollment - no (\%) & $31(74)$ & $30(67)$ \\
\hline APACHE II score at enrollment - mean (SD) & $25.7(9.1)$ & $26.5(9.2)$ \\
\hline SOFA score at enrollment - mean (SD) & $10.1(3.7)$ & $10.2(3.7)$ \\
\hline
\end{tabular}

${ }^{a}$ SD denotes standard deviation, BMI: body mass index, APACHE II: Acute Physiology and Chronic Health Evaluation II, SOFA: Sequential Organ Failure Assessment score

${ }^{b}$ Data missing on 5 patients

${ }^{c}$ Data missing on 8 patients

$d_{\text {Data missing on } 6 \text { to } 9 \text { patients }}$ 
Table 2

\begin{tabular}{|c|c|c|c|c|c|}
\hline \multirow{3}{*}{ 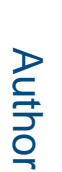 } & Mode of death & & & & \\
\hline & \multirow{2}{*}{ Mode of death } & \multicolumn{2}{|c|}{ All patients $(n=37)$} & \multicolumn{2}{|c|}{ Thiamine deficient $(\mathrm{n}=8)$} \\
\hline & & Thiamine $(\mathrm{n}=19)$ & $\operatorname{Placebo}(n=18)$ & Thiamine $(n=2)$ & Placebo $(n=6)$ \\
\hline 3 & Co-morbid withdrawal of care & $9(47)$ & $6(33)$ & $0(0)$ & $0(0)$ \\
\hline $\bar{c}$ & Refractory Hypotension & $6(32)$ & $10(56)$ & $2(100)$ & $4(67)$ \\
\hline & Respiratory & $1(5)$ & $0(0)$ & $0(0)$ & $0(0)$ \\
\hline & Sudden cardiac arrest & $3(16)$ & $2(11)$ & $0(0)$ & $2(33)$ \\
\hline
\end{tabular}

